\title{
Comparing unbalanced and balanced ratios of blood products in massive transfusion to pediatric trauma patients: effects on mortality and outcomes
}

\author{
Manmeet Sehdev ${ }^{1,3}$ (D) Areg Grigorian ${ }^{1} \cdot$ Catherine Kuza $^{2} \cdot$ Matthew Dolich $^{1} \cdot$ Boris Borazjani $^{1} \cdot$ Michael Lekawa $^{1}$. \\ Jeffry Nahmias ${ }^{1}$
}

Received: 12 February 2020 / Accepted: 5 August 2020 / Published online: 14 August 2020

(c) Springer-Verlag GmbH Germany, part of Springer Nature 2020

\begin{abstract}
Background The utilization and impact of various ratios of transfusions for pediatric trauma patients (PTPs) receiving a massive transfusion (MT) are unknown. Therefore, we sought to determine the risk for mortality in PTPs receiving an MT of $\geq 6$ units of packed red blood cells (PRBC) within $24 \mathrm{~h}$. We compared PRBC: plasma ratio of $>2: 1$ (Unbalanced Ratios, UR) versus $\leq 2: 1$ (Balanced Ratios, BR), hypothesizing decreased risk of mortality with BR.

Methods The Trauma Quality Improvement Program was queried (2014-2016) for PTPs receiving a MT. A multivariable logistic regression model was used to determine risk of mortality.

Results From 239 PTPs receiving an MT, 98 (41\%) received an UR, whereas 141 (59\%) received a BR. The median ratios, respectively, were 2.7:1 and 1.2:1. Compared to BR patients, UR patients had no differences in injury severity score (ISS), hypotension on admission, and intensive care unit stay (all $p>0.05$ ). The mortality rates for BR and UR were similar (46.1\% vs. $52.0 \%, p=0.366)$. Controlling for age, ISS, and severe head injury, UR demonstrated similar risk of mortality compared to $\operatorname{BR}(p=0.276)$. Additionally, $\geq 4: 1$ ratio versus $\leq 2: 1$ showed no difference in associated risk of mortality $(p=0.489)$.

Conclusion In contrast to adult studies, this study demonstrated that MT ratios of $>2: 1$ and even $\geq 4: 1$ were associated with similar mortality compared to BR for PTPs. These results suggest pediatric MT resuscitation may not require strict BR as has been shown beneficial in adult trauma patients. Future prospective studies are needed to evaluate the optimal ratio for PTP MT resuscitation.
\end{abstract}

Level of evidence III; Retrospective Care Management Study.

Keywords Pediatric trauma $\cdot$ Hemorrhage $\cdot$ Massive transfusion $\cdot$ Massive transfusion protocol

Manmeet Sehdev

sehdevm@uci.edu; manmeet.sehdev@ucsf.edu

Areg Grigorian

agrigori@uci.edu

Catherine Kuza

catherine.kuza@med.usc.edu

Matthew Dolich

mdolich@hs.uci.edu

Boris Borazjani

bhborazj@uci.edu

Michael Lekawa

melekawa@hs.uci.edu
Jeffry Nahmias

jnahmias@hs.uci.edu

1 Department of Surgery, Division of Trauma, Burns, Surgical Critical Care, and Acute Care Surgery, University of California, Irvine, Orange, CA, USA

2 Department of Anesthesiology, Keck School of Medicine of the University of Southern California, Los Angeles, CA, USA

3 Irvine Medical Center, University of California, 1001 Health Sciences Rd, Irvine, CA 92617, USA 


\section{Introduction}

According to the Center of Disease \& Control, on average, 12,175 0-to-19-year olds died each year from nonintentional trauma, placing trauma as the first leading cause of death in pediatric patients [1]. Children are capable of maintaining normal blood pressures while suffering up to a $30 \%$ loss of blood volume, yet hemorrhage is still the most preventable form of death in pediatric trauma patients (PTPs), accounting for up to $40 \%$ of all early-traumarelated deaths [2-4]. Coagulopathy has been shown to be an independent predictor of mortality in children [2, 3]. It is estimated that $<2 \%$ of PTPs require a massive transfusion (MT), compared to $3 \%$ of adult trauma patients [5-7]. Furthermore, pediatric patients receiving a MT have been found to have a mortality rate up to $50 \%[2,8-13]$.

A recent survey of 46 hospitals serving pediatric patients in the United States and Canada reported MT activation was at physician discretion and $78 \%$ used a targeted high $(<2: 1)$ packed red blood cells (PRBC): plasma ratio $[5,9]$. This is predominantly based on prior adult studies and guidelines [16-21], such as a prior retrospective military analysis by Borgman et al. showing up to an eight-fold improved mortality with ratios closest to $1: 1$ [18]. However, there are limited pediatric studies regarding the benefit of a balanced resuscitation for PTPs [22, 23, 25-29]. Further confusing this issue is the lack of uniformity with the definition of MT for pediatric trauma. Neff et al. defined MT as $40 \mathrm{ml} / \mathrm{kg}$ for all blood products as an inflection point for identifying critically injured children at high risk for early and in-hospital death [14]. Others have used $>50 \%$ total body volume (TBV) in $3 \mathrm{~h}$ or transfusion $>100 \% \mathrm{TBV}$ in $24 \mathrm{~h}[9,15]$.

Due to the lack of consensus data on the appropriate ratio of MT for pediatric trauma patients, we sought to use a large national database to determine the risk for mortality in PTPs receiving a MT of $\geq 6$ units of PRBC within $24 \mathrm{~h}$. We compared PRBC: plasma ratio of $>2: 1$ (Unbalanced Ratio, UR) versus $\leq 2: 1$ (Balanced Ratio, $\mathrm{BR})$, hypothesizing decreased risk of mortality with a BR.

\section{Methods}

We performed a retrospective database analysis, approved by the institutional review board at the University of California, Irvine. The Trauma Quality Improvement Program (TQIP) database is a national de-identified database backed by the American College of Surgeons with 825 participating trauma centers across the USA. We queried the TQIP database from 2014 to 2016 to identify
PTPs $<18$ years old receiving a MT after trauma. For this study, MT was defined as receiving $\geq 6$ units of PRBC within $24 \mathrm{~h}$. Patients with no documented data on blood product transfusion were excluded. We compared PTPs receiving PRBC: plasma ratio of $>2: 1$ (UR) versus $\leq 2: 1$ (BR). An additional analysis of severely UR of $\geq 4: 1$ PRBC: plasma and BR of $\leq 2: 1$ was also performed. Two additional multivariable regression analyses were performed on subgroups based on age of the patients, one evaluating PTPs 1-to-12 years old and a second evaluating PTPs 13-to-17 years old.

Patient demographic information was collected including age, race, gender, hypotension on admission, and Glasgow Coma Scale (GCS) score. The injury profile included the injury severity score (ISS) and mechanism of injury. Associated injuries included traumatic brain injury (TBI), spine, upper extremity, lower extremity, heart, lung, stomach, small intestine, colorectal, pancreas, liver, spleen, and kidney. These were defined by the appropriate international classification of disease (ICD) version-9-diagnosis codes. The primary outcome was mortality. In addition, we evaluated total hospital length-of-stay (LOS), intensive care unit (ICU) LOS, ventilator days, discharge disposition, and other complications including acute kidney injury (AKI), pneumonia, deep venous thrombosis (DVT), acute respiratory distress syndrome (ARDS), and cardiac arrest.

Descriptive statistics were used to evaluate the study population. A Student's $t$ test or Mann-Whitney $U$ test was used to compare continuous variables and chi-square was used to compare categorical variables for bivariate analysis. Categorical data were reported as percentages, and continuous data were reported as medians with interquartile range or as means with standard deviation. A multivariable stepwise-forward hierarchical logistic regression model was used for analysis. Covariates were chosen based on a review of the literature and included age, ISS $\geq 25$, and severe head injury $[23,25,26,29]$. These variables were included in a hierarchical multivariable logistic regression model and the adjusted risk for mortality was reported with an odds ratio (OR) and $95 \%$ confidence intervals (CI). An additional similar analysis was performed on patients receiving a severely UR, defined as $\geq 4: 1$ ratio of PRBC: plasma. All $p$ values were two-sided, with a statistical significance level of $<0.05$. All analyses were performed with IBM SPSS Statistics for Windows (Version 24, IBM Corp., Armonk, NY).

\section{Results}

\section{Demographics}

From 239 PTPs receiving an MT, 98 (41\%) received an UR, whereas $141(59 \%)$ received a BR. The median ratios 
of PRBC: plasma, respectively, were 2.7:1 and 1.2:1. The median age and IQR were older for PTPs receiving BR versus PTPs receiving UR (14 (6)- vs. 12 (9)-year-old, $p=0.007)$. In addition, the age of the BR group ranged from 1 to 17 years old and the UR group also ranged from 1 to 17 years old. Both groups had a similar percentage of males $(69.5 \%$ vs. $70.4 \%, p=0.910)$. There were no differences in ethnicities (all $p>0.05$ ) and median GCS scores ( 3 vs. $3, p=0.517$ ) between the two groups. There was also no difference in rate of hypotension on admission $(34.8 \%$ vs. $39.1 \%, p=0.513)$ and median ISS (32 vs. 29.50, $p=0.910)$ between PTPs receiving a BR compared to an UR (Table 1).

\section{Risk of mortality}

After adjusting for covariates, there was no difference in associated risk of mortality when comparing patients receiving an UR compared to a BR (OR 1.44, CI 0.75-2.78, $p=0.271)$. The strongest independent variable associated with increased risk of mortality was severe head injury (OR 5.27, CI 2.71-10.2, $p<0.001$ ) followed by ISS $\geq 25$ (OR 2.35 , CI 1.06-5.18, $p=0.035$ ) (Table 2). On a sub-analysis, patients that received severely UR compared to a $\mathrm{BR} \leq 2: 1$ (OR 1.42 CI $0.53-3.80, p=0.489$ ) still had no difference in associated risk of mortality. (See Table 3). The analysis for patients $1-12$ years old $(n=90)$ showed that there was no
Table 1 Demographics of pediatric trauma patients receiving unbalanced ratios of MT resuscitation versus balanced ratios of MT resuscitation

\begin{tabular}{|c|c|c|c|c|}
\hline Characteristic & $\operatorname{MT}(n=239)$ & $\mathrm{BR}(n=141)$ & $\mathrm{UR}(n=98)$ & $p$ value \\
\hline Age, year, median (IQR) & $14(6)$ & $14(6)$ & $12(9)$ & 0.007 \\
\hline ISS, median (IQR) & $35(17)$ & $32(19)$ & $29.50(23)$ & 0.910 \\
\hline Male, $n(\%)$ & $167(69.9 \%)$ & $98(69.5 \%)$ & $69(70.4 \%)$ & 0.881 \\
\hline Teaching-university patients, $n(\%)$ & $178(74.5 \%)$ & $106(75.2 \%)$ & $72(73.5 \%)$ & 0.766 \\
\hline Hypotension on admission, $n(\%)$ & $82(36.6 \%)$ & $46(34.8 \%)$ & $36(39.1 \%)$ & 0.513 \\
\hline Glasgow Coma Scale Score, median (IQR) & & $3(11)$ & $3(12)$ & 0.517 \\
\hline \multicolumn{5}{|l|}{ Ethnicities, $n(\%)$} \\
\hline Black & $66(35.3 \%)$ & $42(38.2 \%)$ & $24(31.2 \%)$ & 0.323 \\
\hline White & $121(50.6 \%)$ & $68(48.2 \%)$ & $53(54.1 \%)$ & 0.373 \\
\hline Asian & $5(4.0 \%)$ & $4(5.6 \%)$ & $1(1.9 \%)$ & 0.292 \\
\hline Hispanic & $40(18.5 \%)$ & $21(16.0 \%)$ & $19(22.4 \%)$ & 0.243 \\
\hline \multicolumn{5}{|l|}{ Specific Mechanisms, $n(\%)$} \\
\hline Bicyclist & $8(3.3 \%)$ & $6(4.3 \%)$ & $2(2.0 \%)$ & 0.349 \\
\hline Blunt mechanism & $105(70.5 \%)$ & $60(67.4 \%)$ & $45(75.0 \%)$ & 0.320 \\
\hline Pedestrian & $18(7.5 \%)$ & $8(5.7 \%)$ & $10(10.2 \%)$ & 0.192 \\
\hline Stab & $6(2.5 \%)$ & $2(1.4 \%)$ & $4(4.1 \%)$ & 0.196 \\
\hline Gunshot Wound & $33(13.8 \%)$ & $24(17.0 \%)$ & $9(9.2 \%)$ & 0.084 \\
\hline Fall & $3(1.3 \%)$ & $3(2.1 \%)$ & $0(0.0 \%)$ & 0.146 \\
\hline Suicide & $5(2.1 \%)$ & $3(2.1 \%)$ & $2(2.0 \%)$ & 0.963 \\
\hline $\mathrm{MCV}$ & $64(26.8 \%)$ & $36(25.5 \%)$ & $28(28.6 \%)$ & 0.602 \\
\hline \multicolumn{5}{|l|}{ Associated Injuries, $n(\%)$} \\
\hline Traumatic Brain Injury & $85(35.6 \%)$ & $50(35.5 \%)$ & $35(35.7 \%)$ & 0.968 \\
\hline Spine fracture & $48(20.1 \%)$ & $31(22.0 \%)$ & $17(17.3 \%)$ & 0.379 \\
\hline Upper extremity fracture & $34(14.2 \%)$ & $23(16.3 \%)$ & $11(11.2 \%)$ & 0.268 \\
\hline Lower extremity fracture & $42(17.6 \%)$ & $28(19.9 \%)$ & $14(14.3 \%)$ & 0.266 \\
\hline Lung & $84(35.1 \%)$ & $50(35.5 \%)$ & $34(34.7 \%)$ & 0.903 \\
\hline Heart & $7(2.9 \%)$ & $4(2.8 \%)$ & $3(3.1 \%)$ & 0.919 \\
\hline Stomach & $6(2.5 \%)$ & $4(2.8 \%)$ & $2(2.0 \%)$ & 0.699 \\
\hline Small intestine & $23(9.6 \%)$ & $17(12.1 \%)$ & $6(6.1 \%)$ & 0.126 \\
\hline Colorectal & $23(9.6 \%)$ & $14(9.9 \%)$ & $9(9.2 \%)$ & 0.848 \\
\hline Pancreas & $7(2.9 \%)$ & $7(5.0 \%)$ & $0(0.0 \%)$ & 0.025 \\
\hline Liver & $42(17.6 \%)$ & $28(19.9 \%)$ & $14(14.3 \%)$ & 0.266 \\
\hline Spleen & $40(16.7 \%)$ & $24(17.0 \%)$ & $16(16.3 \%)$ & 0.887 \\
\hline Kidney & $22(9.2 \%)$ & $11(7.8 \%)$ & $11(11.2 \%)$ & 0.368 \\
\hline
\end{tabular}

$I Q R$ interquartile range, ISS Injury Severity Score, $M V C$ motor vehicle collision, $M T$ massive transfusion, $U R$ unbalanced ratio (> 2:1 PRBC:Plasma), BR balanced ratio ( $\leq 2: 1$ PRBC:Plasma) 
Table 2 Multivariate analysis of risk of mortality in pediatric trauma patients receiving unbalanced ratios of MT resuscitation versus balanced ratios of MT resusciatation, adjusting for age, ISS, and severe head injury

\begin{tabular}{lllr}
\hline Risk factors & Odds ratio & CI & $p$ value \\
\hline UR of MT Resuscitation & 1.44 & $0.75-2.78$ & 0.271 \\
Age & 0.95 & $0.89-1.02$ & 0.174 \\
ISS & 2.35 & $1.06-5.18$ & 0.035 \\
Severe Head Injury & 5.27 & $2.71-10.2$ & $<0.001$ \\
Pancreas Injury & 1.18 & $0.17-8.09$ & 0.867 \\
\hline
\end{tabular}

$C I$ confidence interval, ISS injury severity score, $M T$ massive transfusion, $U R$ unbalanced ratio ( $>2: 1$ PRBC:Plasma), $B R$ balanced Ratio $(\leq 2: 1$ PRBC:Plasma)

Table 3 Multivariable analysis of risk of mortality in pediatric trauma patients receiving unbalanced ratios of MT $(\geq 4: 1)$ versus balanced ratios of MT $(\leq 2: 1)$, adjusting for age, ISS, and severe head injury

\begin{tabular}{lllr}
\hline Risk factors & Odds ratio & CI & $p$ value \\
\hline UR of MT Resuscitation & 1.42 & $0.53-3.80$ & 0.489 \\
Age & 0.97 & $0.88-1.06$ & 0.451 \\
ISS & 3.22 & $1.18-8.82$ & 0.023 \\
Severe Head Injury & 5.02 & $2.32-10.87$ & $<0.001$ \\
\hline
\end{tabular}

$C I$ confidence Interval, ISS injury severity score, $M T$ massive transfusion, $U R$ unbalanced ratio ( $\geq 4: 1$ PRBC:Plasma), $B R$ balanced ratio $(\leq 2: 1$ PRBC:Plasma)

difference in the associated risk of mortality between those receiving BR and UR (OR 1.71, CI 0.65-4.50, $p=0.277$ ). For those aged 13-to-17 years old $(n=149)$, there was again no difference in the associated risk of mortality between those receiving BR and UR (OR 1.58, CI 0.60-4.16, $p=0.351)$.

\section{Clinical outcomes and complications}

When comparing PTPs who received a BR to patients who received an UR, there was no difference in terms of LOS ( 9 vs. 8.5 days, $p=0.900$ ), ICU LOS ( 5 vs. 4 days, $p=0.722$ ), or ventilator days (3 vs. $3, p=0.304$ ). (See Table 4). There was also no difference in all complications including AKI, pneumonia, DVT, ARDS, and cardiac arrest (all $p>0.05$ ). PTPs in the BR group had a mortality rate of $46.1 \%$ compared to $52 \%$ in the UR group $(p=0.366)$. PTPs receiving BR after penetrating trauma had a similar mortality rate compared to those receiving UR $(41.4 \%$ vs $46.7 \%, p=0.737)$. PTPs receiving BR after blunt trauma had a similar mortality rate compared to those receiving UR $(48.3 \%$ vs. $55.6 \%, p=0.464)$. There were no differences in discharge dispositions $(p=0.886)$ between the BR and UR cohorts (Table 4). PTPs in the BR group received a higher median number of units of plasma and platelets within $24 \mathrm{~h}$ compared to the UR group; $(7.27$ vs $3.00, p<0.005)$ and ( 2 vs $1.63, p=0.002$ ). There was no difference in units of PRBC ( 8.7 vs $8.08, p=0.816)$ and cryoprecipitate ( 0.6 vs $0.55, p=0.898$ ) within $24 \mathrm{~h}$ between the cohorts.

\section{Discussion}

While the need for MT in PTPs is relatively uncommon, it is associated with a high mortality rate. Contrary to adult studies, this retrospective analysis spanning two years of national data demonstrates no difference in rate and associated risk of mortality when comparing a BR to UR for PTPs receiving a MT. In addition, there was no difference in LOS, ventilator days and complications between the two cohorts. Finally, even patients who received a severely UR had no difference in associated risk of mortality compared to PTPs who received a $B R$.

The ratio of PRBC to plasma in adult trauma patients has previously been shown to improve mortality. The Prospective Observational Multicenter Major Trauma Transfusion (PROMMTT) trial demonstrated that higher plasma and platelet ratios early in resuscitation were associated with three to four times decreased mortality in patients transfused at least three units of blood products [17]. However, the few pediatric trauma studies that have looked into the use of balanced ratios of MT for PTPs have found mixed results [22-29]. Four studies to date have found no improvement with balanced 1:1 ratios of PRBC: plasma for mortality in children [22-25]. For instance, a retrospective single-center study by Hwu et al. studied 38 children receiving a MT, with a median age of 6 years and demonstrated no significant difference for in-hospital mortality between high $(\leq 2: 1$ PRBC: plasma) and low (>2:1) ratio groups $(45.8 \%$ vs. $64.3 \%$, $p=0.278$ ) [23]. In contrast to our findings and these studies, one retrospective study that utilized two years of TQIP data, looking at 465 PTPs undergoing MT, found $>10 \%$ survival benefit with increased ratios of PRBC: plasma (14\% mortality in low ratio group vs $14 \%$ in medium ratio vs $2 \%$ in high ratio group within $4 \mathrm{~h}$ of admission, $p<0.01$ ) [27]. However, this study replaced missing weight data, which may have introduced bias into their results. Another retrospective analysis by Butler et al., observed 583 PTPs undergoing a low, medium, and high ratio of PRBC: plasma and found a $52 \%$ lower risk of mortality at $24 \mathrm{~h}$ amongst PTPs receiving high transfusion ratios (attributed risk reduction of 0.48 , 95\% confidence interval of 0.39-0.92) [28]. However, this study limited its study population to PTPs $\leq 14$ years old and only evaluated patients who survived greater than $4 \mathrm{~h}$ and chose an endpoint of 24-h mortality. In addition, while these authors used a weight-based definition for MT which would be ideal, unfortunately in TQIP $30 \%$ of weight data were 
Table 4 Clinical outcomes in pediatric trauma patients receiving unbalanced ratios of MT resuscitation versus balanced ratios of MT resuscitation

\begin{tabular}{|c|c|c|c|c|}
\hline Outcomes & $\operatorname{MT}(n=239)$ & $\mathrm{BR}(n=141)$ & $\mathrm{UR}(n=98)$ & $p$ value \\
\hline LOS, days, median (IQR) & & $9(27)$ & $8.5(21)$ & 0.900 \\
\hline ICU, days, median (IQR) & & $5(13)$ & $4(11)$ & 0.722 \\
\hline Ventilator, days, median (IQR) & & $3(5)$ & $3(8)$ & 0.304 \\
\hline \multicolumn{5}{|l|}{ Mortality, $n(\%)$} \\
\hline All mortality & $116(48.5 \%)$ & $65(46.1 \%)$ & $51(52.0 \%)$ & 0.366 \\
\hline Mortality among Blunt trauma & $54(51.4 \%)$ & $29(48.3 \%)$ & $25(55.6 \%)$ & 0.464 \\
\hline Mortality among Penetrative trauma & $19(43.2 \%)$ & $12(41.4 \%)$ & $7(46.7 \%)$ & 0.737 \\
\hline \multicolumn{5}{|l|}{ Blood Products within $24 \mathrm{~h}$, median (units) } \\
\hline PRBC & & $8.7(7)$ & $8.08(6)$ & 0.816 \\
\hline Plasma & & $7.27(6)$ & $3(2)$ & $<0.005$ \\
\hline Platelets & & $2(2.90)$ & $1.63(1.24)$ & 0.002 \\
\hline Cryoprecipitate & & $0.6(1.80)$ & $0.55(0.97)$ & 0.898 \\
\hline \multicolumn{5}{|l|}{ Discharge dispositions, $n(\%)$} \\
\hline Home & $69(56.5 \%)$ & $44(57.9 \%)$ & $25(54.3 \%)$ & 0.886 \\
\hline Long Term Acute Care & $51(41.8 \%)$ & $31(40.8 \%)$ & $20(43.5 \%)$ & 0.886 \\
\hline Skilled Nursing Home & $2(1.6 \%)$ & $1(1.3 \%)$ & $1(2.2 \%)$ & 0.886 \\
\hline \multicolumn{5}{|l|}{ Complications, $n(\%)$} \\
\hline Acute Kidney Injury & $10(4.2 \%)$ & $8(5.7 \%)$ & $2(2.0 \%)$ & 0.168 \\
\hline ARDS & $6(2.5 \%)$ & $4(2.8 \%)$ & $2(2.0 \%)$ & 0.699 \\
\hline Cardiac Arrest & $39(16.3 \%)$ & $20(14.2 \%)$ & $19(19.4 \%)$ & 0.284 \\
\hline Decubitus & $15(6.3 \%)$ & $13(9.2 \%)$ & $2(2.0 \%)$ & 0.024 \\
\hline Deep Infection & $4(1.7 \%)$ & $3(2.1 \%)$ & $1(1.0 \%)$ & 0.512 \\
\hline Deep Venous Thrombosis & $15(6.3 \%)$ & $8(5.7 \%)$ & $7(7.1 \%)$ & 0.645 \\
\hline Compartment Syndrome Extremity & $1(0.4 \%)$ & $0(0.0 \%)$ & $1(1.0 \%)$ & 0.229 \\
\hline Organ Space Infection & $3(1.3 \%)$ & $3(2.1 \%)$ & $0(0.0 \%)$ & 0.146 \\
\hline Pneumonia & $6(2.5 \%)$ & $3(2.1 \%)$ & $3(3.1 \%)$ & 0.650 \\
\hline Pulmonary Embolism & $2(0.8 \%)$ & $0(0.0 \%)$ & $2(2.0 \%)$ & 0.088 \\
\hline CVA & $6(2.5 \%)$ & $5(3.5 \%)$ & $1(1.0 \%)$ & 0.220 \\
\hline Superficial Infection & $4(1.7 \%)$ & $3(2.1 \%)$ & $1(1.0 \%)$ & 0.512 \\
\hline Urinary Tract Infection & $6(2.5 \%)$ & $4(2.8 \%)$ & $2(2.0 \%)$ & 0.699 \\
\hline CRBSI & $2(0.8 \%)$ & $2(1.4 \%)$ & $0(0.0 \%)$ & 0.236 \\
\hline Unplanned OR & $8(0.3 \%)$ & $4(2.8 \%)$ & $4(4.1 \%)$ & 0.599 \\
\hline Unplanned ICU & $4(1.7 \%)$ & $3(2.1 \%)$ & $1(1.0 \%)$ & 0.512 \\
\hline Severe Sepsis & $3(1.3 \%)$ & $2(1.4 \%)$ & $1(1.0 \%)$ & 0.786 \\
\hline CAUTI & $1(0.4 \%)$ & $1(0.7 \%)$ & $0(0.0 \%)$ & 0.403 \\
\hline CLASBI & $1(0.4 \%)$ & $1(0.7 \%)$ & $0(0.0 \%)$ & 0.403 \\
\hline
\end{tabular}

$I Q R$ interquartile range, $L O S$ length-of-stay, $I C U$ intensive care unit, $M T$ massive transfusion, $U R$ unbalanced ratio ( $>2: 1$ PRBC:Plasma), $B R$ balanced ratio ( $\leq 2: 1$ PRBC:Plasma), ARDS acute respiratory distress syndrome, $C V A$ Cerebrovascular accident, CRBSI Catheter-related bloodstream infection, OR Operating room, CAUTI Catheter-associated urinary tract infection, CLASBI central line-associated bloodstream infection. missing. In contrast, our study would include the child in the "golden hour" of hemorrhage control who dies, as well as incorporate the true endpoint for success which would be the child surviving to leave the hospital. Our study also uniquely adds further insight into this issue by demonstrating that even a severely unbalanced ratio ( $\geq 4: 1$ PRBC to plasma) was associated with similar risk of mortality compared to a BR. In addition, even on subgroup analysis both teenagers and children age 1-12 years old had similar findings.
Although the younger cohort was a relatively small population and may be subject to a type II error. Possible explanations for these findings include that some of these studies contained large variations in the mechanisms of injuries, as seen with the military setting studies demonstrating higher rates of blast and penetrating injuries. In addition, one study excluded PTPs with severe head injury [25], while another excluded PTPs who died within the first $24 \mathrm{~h}$, thus excluding patients who died from exsanguination [22]. When 
comparing adult data from the PROMMT study, median ISS of 26 and median GCS 13, and PROPPR trial, ISS of 26.5 vs. 26 in the randomized 1:1 vs. 1:2 groups and median GCS of 14 in both groups, these adult trauma patients were severely injured similar to the pediatric patients in this study $[17,18]$. However, this study controlled for severe head injury understanding in a pediatric population this is significantly associated with mortality and was different then the aforementioned adult studies [25, 26]. Also, there is no standardization of the definition of MT for PTPs [30-32]. Most studies defined MT using $\mathrm{a} \geq 40 \mathrm{ml} / \mathrm{kg}$ of PRBC or TBV [14, 23, 28], while others employed $\geq 70-80 \mathrm{ml} / \mathrm{kg}$ or $>50 \%$ total blood volume $[25,26]$. Our study utilized a definition of $\geq 6$ units of PRBC within $24 \mathrm{~h}$ due to limitations of the database (i.e. incomplete weight data available). This definition may have missed some infants and small children, however, using this data point certainly captured PTPs receiving even larger transfusions. Future randomized prospective trials are greatly needed to further assess the benefit of BR to mortality in PTPs.

MT is an important intervention for exsanguination but is not without its own infectious and non-infectious risks. MT can also cause volume overload, electrolyte and metabolic disturbances, such as hypokalemia and hypocalcemia, and dilution coagulopathy [12]. However, a majority of the pediatric trauma retrospective analyses have shown no difference in rates of complications between their balanced and unbalanced groups [22-25, 27]. For example, Cunningham et al. demonstrated no difference in ventilator free days (14 vs 11 vs $12, p=0.87$ ), hospital-free days ( 0 vs 0 vs $0, p=0.91$ ), and ICU-free days ( 7 vs 9 vs $8, p=0.92$ ) between their three cohorts of low ( $>2: 1$ PRBC: plasma), medium $(\leq 2: 1$ to $<1: 1)$, and high $(\leq 1: 1)$ [27]. Similarly, we found no difference in ventilator days, LOS, and ICU LOS. When comparing patients who received a BR to PTPs who received an UR in our study, there was also no difference in rates of complications including AKI, pneumonia, DVT, ARDS, or cardiac arrest. This has also been shown in other pediatric trauma studies [27, 28]. Similarly, an adult pragmatic randomized clinical trial, by Holcomb et al., found no significant differences with AKI, pneumonia, ARDS, or cardiac arrest between ratios 1:1:1 vs. 2:1:1 of PRBC: platelets: plasma [16]. Prior studies have demonstrated age-related changes in hemostasis and thrombosis in pediatric physiology $[4,10$, $12,32,34-36]$. A possible physiologic explanation for this difference seen between adults and children can be attributed to levels of pro-coagulants, such as Vitamin-K-dependent coagulation factors, that remain decreased throughout childhood [34]. In addition, children have decreased rates of failure of non-operative management of solid organ injuries [35, 36], further suggesting improved hemostatic capabilities. This suggests that the ratio of resuscitation is not related to the occurrence of these complications and provides further evidence that strict adherence to BR for PTPs receiving a MT may not be indicated.

Our study has many limitations, including those inherent to a retrospective database study, such as misclassification, reporting bias and missing data. In addition, missing pertinent variables include time to hemorrhage control, laboratory values related to coagulopathy, and the specific causes of death and/or whether it was related to hemorrhage. A lack of power creating a Type II error is also possible. The determination for using $24 \mathrm{~h}$ was arbitrary, as this database lacks documentation of the exact time hemostasis was achieved, which was utilized in a prior adult study [16] and, thus, this study may reflect time beyond the point where hemostasis was achieved, and thereby introduce bias. Another significant limitation to our study is that TQIP only had weight data for seventy percent of patients, thus not allowing us to utilize a weight-based definition of MT, which would be ideal to evaluate our findings using this definition of MT as well. Additionally, we are missing data regarding the time to receiving transfusion, the order of the blood products received and whether institutions had specific MT protocols and/or access to viscoelastic assays, which in the adult population have been demonstrated to improve mortality [33].

\section{Conclusion}

The rate of mortality overall for PTPs receiving a MT was nearly $50 \%$. In contrast to adult studies, in this national database analysis, we found there was no difference in rate or associated risk of mortality for PTPs receiving a BR compared to those receiving an UR. Also, PTPs receiving an UR had similar rates of complications and LOS compared to PTPs receiving a BR. In addition, there was no difference in associated risk of mortality for PTPs receiving a severely UR. Continued work on developing future prospective studies is needed and will help elucidate the optimal ratio of PRBC: plasma for PTP MT resuscitations.

Author contributions MS: Literature search, writing, literature search, data interpretation, critical revision. AG: Study design, data collection, statistics, data analysis, data interpretation, critical revision. CK: Data analysis, data interpretation, critical revision. MD: Data analysis, data interpretation, critical revision. BB: Data analysis, data interpretation, critical revision. ML: Data analysis, data interpretation, critical revision. JN: Study design, data analysis, data interpretation, critical revision.

Funding No funding was received for this work.

\section{Compliance with ethical standards}

Conflict of interest The authors declare that they have no conflicts of interest. 
Human and animal rights statement This research involved humans. However, since this retrospective study was performed using a national database with de-identified patients, risk to participants is minimal. There is no consent required.

\section{References}

1. Borse NN, Gilchrist J, Dellinger AM, Rudd RA, Ballesteros MF, Sleet DA. CDC Childhood Injury Report: Patterns of Unintentional Injuries among 0-19 Year Olds in the United States, 2000 2006. Atlanta (GA): Centers for Disease Control and Prevention, National Center for Injury Prevention and Control; 2008.

2. Hendrickson JE, Shaz BH, Pereira G, Atkins E, Johnson KK, Bao G, Easley KA, Josephson CD. Coagulopathy is prevalent and associated with adverse outcomes in transfused pediatric trauma patients. J Pediatr. 2012;160:204e-9e3.

3. Patregnani JT, Borgman MA, Maegele M, Wade CE, Blackbourne LH, Spinella PC. Coagulopathy and shock on admission is associated with mortality for children with traumatic injuries at combat support hospitals. Pediatr Crit Care Med. 2011;3:273-7.

4. Christiaans S, Duhacheck-Stapelman A, Russell R, Lisco SJ, Kerby JD, Pittet JF. Coagulopathy after severe pediatric trauma: A review. Shock. 2014;41(6):476-90.

5. Livingston MH, Singh S, Merritt NH. Massive transfusion in paediatric and adolescent trauma patients: incidence, patient profile, and outcomes prior to a massive transfusion protocol. Injury Int J. Cared Injured. 2014;45:1301-6.

6. Blain S, Paterson N. Pediatric massive transfusion. BJA Educ. 2015;16(8):269-75.

7. Shroyer MC, Griffin RL, Mortellaro VE, Russell TR. Massive transfusion in pediatric trauma: analysis of the National Trauma Databank. J Surg Res. 2017;208:166-72.

8. Hwu R, Spinella P, Keller MS, Baker D, Wallendorf M, Leonard JC. The effect of massive transfusion protocol implementation on pediatric trauma care. Transfusion. 2016;56(56):2712-9.

9. Horst J, Leonard JC, Vogel A, Jacobs R, Spinella PC. A survey of US and Canadian hospitals' paediatric massive transfusion protocol policies. Transfus Med. 2016;26(1):49-56.

10. Chidester SJ, Williams N, Wang W, Groner JI. A pediatric massive transfusion protocol. J Trauma Acute Care Surg. 2012;73:1273-7.

11. Diab Y, Wong E, Luban N. Massive transfusion in children and neonates. Br J Haematol. 2013;161:15-26.

12. Kamyszek RW, Leraas HJ, Reed C, Ray CM, Nag UP, Poisson JL, Tracy ET. Massive transfusion in the pediatric population: A systemic review and summary of best-evidence practice strategies. J Trauma Acute Care Surg 2019. 86(4)

13. Dehmer JJ, Adamson WT. Massive transfusion and blood product use in the pediatric trauma patient. Semin Pediatr Surg. 2010;19:286-91

14. Neff LP, Cannon JW, Morrison JJ, Edwards MJ, Spinella PC, Borgman MA. Clearly defining pediatric massive transfusion: cutting through the fog and friction with combat data. J Trauma Acute Care Surg. 2015;78(1):22-8.

15. Drucker NA, Wang SK, Newton C. Pediatric trauma-related coagulopathy: balanced resuscitation, goal-directed therapy and viscoelastic assays. Semin Pediatr Surg. 2019;28:61-6.

16. Holcomb JB, Tiley BC, Baraniuk S, Fox EE, Wade CE, Podbielski JM, del Junco DJ, Brasel JK, Vulger EM, Callcut RA, et al. Transfusion of plasma, platelets, and red blood cells in a 1:1:1 vs a 1:1:2 ratio and mortality in patients with severe trauma. JAMA. 2015;313(5):471-82.

17. Holcomb JB, del Junco DJ, Fox EE, Wade CE, Cohen MJ, Schreiber MA, Alarcon LH, Bai Y, Brasel KJ, Bulger EM, et al.
The Prospective, Observational, Multicenter, Major Trauma Transfusion (PROMMTT) study: comparative effectiveness of a time-varying treatment with competing risks. JAMA Surg. 2013;148:127-36.

18. Borgman MA, Spinella PC, Perkins JG, Grathwohl KW, Repine T, Beekley AC, Sabesta J, Jenkins D, Wade CE, Holcomb JB. The ratio of blood products transfused affects mortality in patients receiving massive transfusions at a combat support hospital. J Trauma. 2007;63:805-13.

19. Spinella P, Wade C, Blackbourne LH, Borgman MA, Zarzabal LA, Du F, Perkins JG, Maegele M, Schreiber M, Hess JR, et al. The association of blood component use ratios with the survival of massively transfused trauma patients with and without severe brain injury. J Trauma. 2011;71:S343-S352352.

20. Nunns G, Moore E, Stettler GR, Moore HB, Ghasabyan A, Cohen M, Huebner BR, Sillman CC, Banerjee A, Sauaia A. Empiric transfusion strategies during life-threatening hemorrhage. Surgery. 2018;164:306-11.

21. Wang $\mathrm{CH}$, Hsieh WH, Chou HC, Huang YS, Shen JH, Yeo YH, Chang HE, Chen SC, Lee CC. Liberal versus restricted fluid resuscitation strategies in trauma patients: a systemic review and metaanalysis of randomized control trials and observational studies. Crit Care Med. 2014;42(4):954-61.

22. Nosanov L, Inaba K, Okoye O, Resnick S, Upperman J, Shulman I, Rhee P, Demetriades D. The impact of blood product ratios in massively transfused pediatric trauma patients. Am J Surg. 2013;206:655-60.

23. Hwu RS, Keller MS, Spinella PC, Baker D, Tao Y, Leonard JC. Potential effects of high plasma to red blood cell ratio transfusion in pediatric trauma. Trauma. 2017;19(1):21-7.

24. Hendrickson JE, Shaz BH, Pereira G, Parker PM, Jessup P, Atwell F, Polstra B, Atkins E, Johnson KK, Bao G, Easley KA, Josephson CD. Implementation of a pediatric trauma massive transfusion protocol: one institution's experience. Transfusion. 2012;52:1228-366.

25. Cannon JW, Johnson MA, Caskey RC, Borgman MA, Neff LP. High ratio plasma resuscitation does not improve survival in pediatric trauma patients. J Trauma Acute Care Surg. 2017;83(2):211-7.

26. Edwards MJ, Lustik MB, Clark ME, Creamer KM, Tuggle D. The effects of balanced blood component resuscitation and crystalloid administration in pediatric trauma patients requiring transfusion in Afghanistan and Iraq 2002 to 2012. J Trauma Acute Care Surg. 2015;78:330-5.

27. Cunningham E, Rosenfeld E, Zhu H, Naik-Mathuria BJ, Russel RT, Vogel AM. A high ratio of plasma:pRBC improves survival in massively transfused injured children. J Surg Res 2019(233)13e220

28. Butler EK, Mills BM, Arbabi S, Bulger EM, Vavilala MS, Groner JI, Stansbury LG, Hess JR, Rivara FP. Association of blood component ratios with 24-hour mortality in injured children receiving massive transfusion. Pediatric Critical Care. 2019;47(7):975-83.

29. Noland DK, Apelt N, Greenwell C, Tweed J, Notrica DM, Garcia NM, Maxson RT, Eubanks JW III, Alder AC. Massive transfusion in pediatric trauma: ATOMAC perspective. J Pediatr Surg. 2019;54:345-9.

30. Karam O, Tucci M. Massive transfusion in children. Transfus Med Rev. 2016;30:213-6.

31. Maw G, Furyk C. Pediatric massive transfusion a systemic review. Pediatr Emer Care. 2018;34:594-8.

32. Nystrup K, Stensballe BM, Johansson PI, Ostrowski SR. Transfusion therapy in paediatric trauma patients: a review of the literature. Scand J Trauma Resusc Emerg Med. 2015;23:21.

33. Gonzalez E, Moore EE, Moore HB, Chapman MP, Chin TL, Ghasabyan A, Wohlauer MV, Barnett CC, Bensard DD, Biffl WL, et al. Goal-directed hemostatic resuscitation of trauma-induced 
coagulopathy: a pragmatic randomized clinical trial comparing a viscoelastic assay to conventional coagulation assays. Ann Surg. 2006;263(6):1051-9.

34. Kuhle S, Male C, Mitchell L. Developmental hemostasis: pro- and anticoagulant systems during childhood. Semin Thromb Hemost. 2003;29(4):329-38. https://doi.org/10.1055/s-2003-42584.

35. Ozturk H, Dokucu AI, Onen A, Otçu S, Gedik S, Azal OF. Non-operative management of isolated solid organ injuries due to blunt abdominal trauma in children: a fifteen-year experience. Eur J Pediatr Surg. 2004;14(1):29-34. https://doi. org/10.1055/s-2004-815777.

36. Daodu O, Brindle M, Alvarez-Allende CR, Lockyer L, Weber B, Lopushinsky SR. Outcomes of an accelerated care pathway for pediatric blunt solid organ injuries in a public healthcare system. J Pediatr Surg. 2017;52(5):826-31. https://doi.org/10.1016/j.jpeds urg.2017.01.037. 